\title{
Results of beetles (Coleoptera) survey of Zástudánčí National Nature Reserve (Central Moravia) 2008 - part 1
}

\section{Oto Nakládal}

Results of beetles (Coleoptera) survey of Zástudánčí National Nature Reserve (Central Moravia) 2008 - part 1.- Čas. Slez. Muz. Opava (A), 60: 63-78, 2011.

\begin{abstract}
During the beetles survey of Zástudánčí National Nature Reserve (Central Moravia), 3585 specimens belonging to 857 species from 64 families were recorded. Faunistic data of 23 of them (Haliplidae, Dytiscidae, Carabidae, Hydrophilidae, Histeridae, Hydraenidae, Ptiliidae, Leiodidae, Scydmaenidae, Silphidae, Staphylinidae, Lucanidae, Geotrupidae, Scarabaeidae, Buprestidae, Cerophytidae, Eucnemidae, Throscidae, Elateridae, Drilidae, Lycidae, Lampyridae, and Cantharidae) are presented. Presenting data represent 2191 specimens belonging to 456 species. Critically endangered species (according to Red list of theatened species in the Czech Republic - Invertebrates) Negastrius pulchellus and N. sabulicola (Elateridae) and Cerophytum elateroides (Cerophytidae) were recorded.

Key words: faunistics, Coleoptera, Zástudánčí National Nature Reserve, Czech Republic, Negastrius pulchellus, Negastrius sabulicola, Cerophytum elateroides
\end{abstract}

\section{Introduction}

Zástudánčí National Nature Reserve is very close to nature territory. Despite of this fact was very insufficiently documented. The aim of this survey was to make the most complete list of presenting beetles species for nature preserving purposes.

Inventory survey of aquatic beetles was made by Merta (2004), butterflyes with day activity by Čelechvoský (1993, 2001a,b) and Čelechovská (2001). First integral data about beetles (Coleoptera) show this work untill now. This paper is only the first part of the survey results. Second part will be only published.

\section{Characteristic of the area}

Zástudánčí National Nature Reserve was established already in year 1952. It is situated $4 \mathrm{~km}$ south-easter direction from Tovačov town closely below the Morava and Bečva rivers confluence. It is close-natural flooded forest along Morava river with natural river dynamic. The studied territory is at altitudes of 195-200 m a.s.1. (Šafár et al. 2003) wih area of 100.64 ha. It belongs to the T2 climate region (warm, slightly moist region with characteristics slight winter) (Šafár et al. 2003). Subsoil is formed by Holocene river alluvium. Dominant soil type is gley fluvisol (Šafár et al. 2003). Wide wold of Morava river forms Axis of the Zástudánčí NNR.

The majority of the floodplain area is covered mainly by hardwood forests (association Ficario-Ulmetum campestris). There are much fewer softwood trees and they line the banks of the Morava river. These habitats are represented by associations Fraxino-Populnetum and Salicetum albae. The channel of the Morava river is undisturbed along its whole length of the studied area hence it forms numerous different succession biotopes (e.g. swamps, gravel and/or sand banks which are affected by fluctuating water levels and overgrown by vegetation to various extent).

Survey territory covers two faunistics squares as 6569 and 6669 (Pruner \& Míka 1996). Geographic coordinates (GPS) of the core of NNR is $49^{\circ} 24^{\prime} 14^{\prime \prime N}$; $17^{\circ} 19^{\prime} 3^{\prime \prime} \mathrm{E}$.

\section{Material and methods}

The aim of this survey was to record as many species as possible and hence various collecting methods were utilized, including individual sampling (especially in the riparian zone of the Morava river and aquatic habitats), beating of trees and bushes, sweeping (in day and also at night), capturing of aquatic beetles by sieve, pitfall traps, examination of excrements and carrion, sifting of organic substrates (especially from anthills and tree hollows and bases), night sampling from trees, luring by light on white cloth, capturing on fermented tree sap, luring by ferment fruits, collecting trunks and branches and their subsequent getting unter laboratory contidion.

The pitfall traps (ethylenglycole was utilized to fix the specimens) were set on 29.v.-11.vii.2008 and 11.vii.-17.viii.2008 (to the 4 different biotopes - see the Fig. 1, ten pitfall traps were set on each biotopes) and visited on 9.v., 29.v., 14.vi., 22.vi., 11.vii., 17.viii. and 19.x.2008. 
The nomenclature follows Löbel \& Smetana $(2003,2004,2006,2007)$. The species within each family are listed alphabetically.

\section{Identification}

Family Dytiscidae and Haliplidae identified Jiř́ Hájek (National Museum, Prague), Hydrophilidae and Hydraenidae Martin Fikáček (National Museum, Prague), Histeridae, Eucnemidae and Throscidae Jiř́ Vávra (Ostrava Museum, Ostrava), Leiodidae: Cholevinae Jan Růžička (Czech University of Life Sciences Prague, Prague), Leiodidae: Leiodinae Zdeněk Švec (Prague), Staphylinidae (except Pselaphinae) Pavel Krásenský (Chomutov), Staphylinidae: Pselaphinae and Scydmaenidae Stanislav Vít (Geneva, Switzerland), Scarabaeidae Radek Červenka (Prague), Buprestidae Vít Kubáň (National Museum, Prague), Elateridae Bořivoj Zbuzek (Prague), Cantharidae Vladimír Švihla (National Museum, Prague), others families were identified by author unless otherwise stated in the Results.

\section{Results}

Faunistics data for 23 families are presented in this part of paper (Haliplidae, Dytiscidae, Carabidae, Hydrophilidae, Histeridae, Hydraenidae, Ptiliidae, Leiodidae, Scydmaenidae, Silphidae, Staphylinidae, Lucanidae, Geotrupidae, Scarabaeidae, Buprestidae, Cerophytidae, Eucnemidae, Throscidae, Elateridae, Drilidae, Lycidae, Lampyridae and Cantharidae).

The families contain records of 2191 speciment representing 456 species (from all count of $3585 \mathrm{spec}$. belonging to 857 species from 64 families).

Data „Tovačov env., Zástudánčí National Nature Reserve“ belong to all recorded specimens.

All specimen were captured by the author and they are deposited in his private collection (unless otherwise stated in the Results).

Abbreviations: PFT - pitfall trap

\section{HALIPLIDAE}

Haliplus laminatus (Schaller, 1783): (6569), 9.v.2008, 1 spec.

\section{DYTISCIDAE}

Hydroglyphus geminus (Fabricius, 1792): (6569), 9.v.2008, 2 spec.; (6569), 11.vii.2008, on light, 1 spec.

Hydroporus incognitus Sharp, 1869: (6569), 9.v.2008, 1 spec.

Rhantus suturalis (MacLeay, 1825): (6569), 9.v.2008, 4 spec.

\section{CARABIDAE}

Abax parallelepipedus (Piller et Mitterpacher, 1783): (6569), 11.vii.2008, 5 spec.; (6569), 11.vii.2008, 1 spec.; (6569), 11.vii.-17.viii.2008, PFT - locality 1, 2 spec.; (6569), 11.vii.-17.viii.2008, PFT - locality 4, 1 spec.; (6569), 29.v.-11.vii.2008, PFT - locality 1, 7 spec.; (6569), 29.v.-11.vii.2008, PFT - locality 3, 11 spec.

Abax parallelus (Duftschmid, 1812): (6569), 11.vii.2008, 1 spec.; (6569), 29.v.-11.vii.2008, PFT - locality 1, 6 spec.; (6569), 29.v.-11.vii.2008, PFT - locality 3, 1 spec.; (6569), 29.v.-11.vii.2008, PFT - locality 4, 1 spec.; (6569), 11.vii.-17.viii.2008, PFT - locality 1, 1 spec.; (6569), 11.vii.-17.viii.2008, PFT - locality 2, 4 spec.; (6569), 11.vii.-17.viii.2008, PFT - locality 3, 1 spec.; (6569), 11.vii.-17.viii.2008, PFT - locality 4, 2 spec.

Acupalpus exiguus Dejean, 1829: (6569), 11.vii.2008, on light, 1 spec., P. Veselý det.

Acupalpus flavicollis (Sturm, 1825): (6569), 11.vii.2008, on light, 1 spec.

Acupalpus luteatus (Duftschmid, 1812): (6569), 11.vii.2008, on light, 1 spec.

Acupalpus parvulus (Sturm, 1825): (6569), 11.vii.2008, on light, 1 spec.

Agonum afrum (Duftschmid, 1812): (6569), 14.vi.2008, 2 spec., P. Veselý det.

Agonum gisellae Csiki 1931: (6669), 9.v.2008, 2 spec., P. Veselý det.

Agonum lugens (Duftschmid, 1812): (6569), 11.vii.2008, 3 spec., P. Veselý det.

Agonum marginatum (Linnaeus, 1758): (6669), 9.v.2008, 3 spec.

Agonum viduum (Panzer, 1796): (6569), 29.v.2008, 1 spec., P. Veselý det.

Amara aenea (De Geer, 1774): (6569), 29.v.-11.vii.2008, PFT - locality 3, 1 spec.

Amara aulica (Panzer, 1796): (6569), 17.viii.2008, 2 spec. 
Amara bifrons (Gyllenhal, 1810): (6569), 17.viii.2008, 1 spec.

Amara convexior Stephens, 1828: (6569), 29.v.-11.vii.2008, PFT - locality 3, 2 spec.

Amara familiaris (Duftschmid, 1812): (6569), 29.v.-11.vii.2008, PFT - locality 1, 1 spec.; (6569), 17.viii.2008, 1 spec.

Amara gebleri Dejean, 1831: (6569), 19.x.2008, 1 spec.

Amara plebeja (Gyllenhal, 1810): (6569), 11.vii.2008, 1 spec.; (6569), 11.vii.-17.viii.2008, PFT - locality 3, 1 spec., P. Veselý det.; (6569), 17.viii.2008, 5 spec.

Amara similata (Gyllenhal, 1810): (6569), 17.viii.2008, 1 spec.

Anchomenus dorsalis (Pontoppidan, 1763): (6569), 29.v.-11.vii.2008, PFT - locality 3, 4 spec.; (6569), 11.vii.17.viii.2008, PFT - locality 3, 3 spec.

Anisodactylus binotatus (Fabricius, 1787): (6569), 14.vi.2008, 2 spec.; (6669), 22.vi.2008, 1 spec.

Anisodactylus signatus (Panzer, 1796): (6569), 17.viii.2008, 1 spec.; (6669), 9.v.2008, 1 spec.

Anthracus consputus (Duftschmid, 1812): (6569), 11.vii.2008, on light, 1 spec.

Asaphidion flavipes (Linnaeus, 1761): (6569), 29.v.-11.vii.2008, PFT - locality 3, 1 spec.; (6569), 14.vi.2008, 3 spec.

Badister bullatus (Schrank, 1798): (6569), 11.vii.2008, on light, 1 spec.

Bembidion articulatum (Panzer, 1796): (6569), 29.v.2008, 3 spec.; (6569), 14.vi.2008, 5 spec.; (6569), 22.vi.2008, 2 spec.

Bembidion azurescens azurescens Dalla Torre, 1877: (6569), 29.v.2008, 7 spec.; (6569), 14.vi.2008, 3 spec.; (6569), 22.vi.2008, 12 spec.

Bembidion biguttatum (Fabricius, 1779): (6569), 11.vii.2008, on light, 1 spec.

Bembidion decorum decorum (Panzer, 1799): (6569), 22.vi.2008, 1 spec.

Bembidion dentellum (Thunberg, 1787): (6569), 29.v.2008, 2 spec.; (6569), 14.vi.2008, 1 spec.

Bembidion femoratum femoratum Sturm, 1825: (6569), 17.viii.2008, 1 spec., P. Veselý det.

Bembidion fluviatile Dejean, 1831: (6569), 22.vi.2008, 5 spec.

Bembidion lampros (Herbst, 1784): (6569), 17.viii.2008, 2 spec.; (6569), 19.x.2008, 1 spec.

Bembidion lunulatum (Geoffrey, 1785): (6569), 9.v.2008, 1 spec.

Bembidion modestum (Fabricius, 1801): (6569), 29.v.2008, 5 spec.; (6569), 14.vi.2008, 1 spec.; (6569), 22.vi.2008, 1 spec.

Bembidion monticola Sturm, 1825: (6569), 29.v.2008, 1 spec., P. Veselý det.

Bembidion octomaculatum (Goeze, 1777): (6569), 22.vi.2008, 1 spec.

Bembidion properans (Stephens, 1828): (6569), 9.v.2008, 1 spec.; (6569), 17.viii.2008, 1 spec.

Bembidion punctulatum Drapiez, 1820: (6569), 14.vi.2008, 1 spec.; (6569), 29.v.2008, 1 spec.

Bembidion quadrimaculatum quadrimaculatum (Linnaeus, 1761): (6569), 29.v.2008, 2 spec.; (6569), 14.vi.2008, 3 spec.; (6569), 22.vi.2008, 2 spec.; (6569), 17.viii.2008, 1 spec.

Bembidion semipunctatum (Donovan, 1806): (6569), 29.v.-11.vii.2008, 9 spec., P. Veselý det.; (6569), 14.vi.2008, 5 spec.; (6569), 22.vi.2008, 2 spec.

Bembidion schueppelii Dejean, 1831: (6569), 14.vi.2008, 8 spec.

Bembidion testaceum testaceum (Duftschmid, 1812): (6569), 29.v.2008, 30 spec.; (6569), 14.vi.2008, 1 spec.

Bembidion tetracolum Say, 1823: (6569), 29.v.2008, 1 spec.; (6569), 14.vi.2008, 2 spec.

Bembidion varium (Olivier, 1795): (6569), 9.v.2008, 1 spec.; (6569), 22.vi.2008, 1 spec.

Blemus discus (Fabricius, 1792): (6569), 11.vii.2008, on light, 3 spec.

Brachinus explodens Duftschmid, 1812: (6569), 11.vii.-17.viii.2008, PFT - locality 3, 1 spec., P. Veselý det.; (6569), 17.viii.2008, 1 spec.

Calathus fuscipes (Goeze, 1777): (6569), 29.v.-11.vii.2008, PFT - locality 3, 3 spec.; (6569), 11.vii.17.viii.2008, PFT - locality 3, 3 spec.

Carabus coriaceus Linnaeus, 1758: (6569), 29.v.-11.vii.2008, PFT - locality 1, 1 spec.; (6569), 29.v.11.vii.2008, PFT - locality 3, 2 spec.; (6569), 11.vii.-17.viii.2008, PFT - locality 1, 2 spec.; (6569), 11.vii.17.viii.2008, PFT - locality 3,4 spec.

Carabus granulatus Linnaeus, 1758: (6569), 29.v.-11.vii.2008, PFT - locality 2, 1 spec.; (6569), 29.v.11.vii.2008, PFT - locality 4, 3 spec.; (6569), 11.vii.-17.viii.2008, PFT - locality 4, 1 spec.

Carabus scheidleri Panzer, 1799: (6569), 29.v.-11.vii.2008, PFT - locality 3, 22 spec.; (6569), 29.v.11.vii.2008, PFT - locality 4, 2 spec.; (6569), 11.vii.-17.viii.2008, PFT - locality 3, 2 spec.; (6569), 11.vii.17.viii.2008, PFT - locality 4,1 spec.

Carabus ullrichii Germar, 1824: (6569), 29.v.-11.vii.2008, PFT - locality 1, 1 spec.; (6569), 29.v.-11.vii.2008, PFT - locality 2, 1 spec.; (6569), 29.v.-11.vii.2008, PFT - locality 3, 76 spec.; (6569), 29.v.-11.vii.2008, PFT - locality 4, 1 spec.; (6569), 14. vi. 2008, 7 spec.; (6569), 11.vii.-17.viii.2008, PFT - locality 2, 2 spec.; (6569), 11.vii.-17.viii.2008, PFT - locality 3, 3 spec.; (6569), 11.vii.-17.viii.2008, PFT - locality 4, 2 spec.

Cicindela arenaria viennensis Schrank, 1781: (6569), 22.vi.2008, 4 spec. 
Cicindela hybrida Linnaeus, 1758: (6569), 22.vi.2008, 2 spec.

Clivina collaris (Herbst, 1784): (6569), 29.v.2008, 5 spec.; (6569), 14.vi.2008, 1 spec.

Clivina fossor (Linnaeus, 1758): (6569), 9.v.2008, 1 spec.

Demetrias atricapillus (Linnaeus, 1758): (6569), 17.viii.2008, $3 \mathrm{spec}$.

Diachromus germanus (Linnaeus, 1758): (6569), 9.v.2008, 1 spec.

Dolichus halensis (Schaller, 1783): (6569), 11.vii.2008, on light, 1 spec.

Drypta dentata (Rossi, 1790): (6569), 17.viii.2008, 1 spec.

Dyschirius intermedius Putzeys, 1846: (6569), 29.v. 2008, 1 spec., P. Bulirsch det.

Dyschirius nitidus (Dejean, 1825): (6569), 22.vi.2008, 1 spec., P. Veselý det.

Dyschirius tristis Stephens, 1827: (6569), 14.vi.2008, 2 spec., P. Veselý det.; (6569), 22.vi.2008, 1 spec., P. Veselý det.

Elaphropus diabrachys (Kolenati, 1845): (6569), 22.vi.2008, 1 spec.

Elaphrus aureus P. W. J. Müller, 1821: (6569), 14.vi.2008, 1 spec.

Elaphrus cupreus Duftschmidt, 1812: (6569), 14.vi.2008, 1 spec.

Elaphrus riparius (Linnaeus, 1758): (6569), 29.v.2008, 5 spec.; (6569), 14.vi.2008, 1 spec.

Europhilus micans (Nicolai, 1822): (6569), 29.v.2008, 1 spec.; (6569), 14.vi.2008, 2 spec.; (6569), 14.vi.2008, 4 spec., P. Veselý det.; (6569), 11.vii.2008, on light, 1 spec.; (6569), 11.vii.2008, 1 spec.

Harpalus affinis (Schrank, 1781): (6569), 17.viii.2008, $1 \mathrm{spec.}$

Harpalus atratus Latreille, 1804: (6569), 29.v.-11.vii.2008, PFT - locality 1, 12 spec.; (6569), 14.vi.2008, 1 spec.; (6569), 11.vii.-17.viii.2008, PFT - locality 1, 1 spec.

Harpalus luteicornis (Duftschmid, 1812): (6569), 29.v.-11.vii.2008, PFT - locality 3, 1 spec.

Harpalus tardus (Panzer, 1796): (6569), 29.v.-11.vii.2008, PFT - locality 3, 3 spec.; (6569), 11.vii.17.viii.2008, PFT - locality 3, 1 spec., P. Veselý det.

Chlaenius nigricornis (Fabricius, 1787): (6569), 14.vi.2008, 1 spec.

Chlaenius nitidulus (Schrank, 1781): (6569), 22.vi.2008, 1 spec.

Chlaenius tristis (Schaller, 1783): (6569), 11.vii.2008, on light, 1 spec.

Chlaenius vestitus (Paykull, 1790): (6569), 22.vi.2008, 1 spec.

Leistus rufomarginatus (Duftschmid, 1812): (6569), 29.v.-11.vii.2008, PFT - locality 1, 8 spec.

Leistus terminatus (Panzer, 1793): (6569), 9.v.2008, 1 spec.

Lionychus quadrillum (Duftschmid, 1812): (6569), 14.vi.2008, 3 spec.; (6569), 17.viii.2008, 2 spec.

Loricera pilicornis pilicornis (Fabricius, 1775): (6569), 9.v.2008, 1 spec.

Microlestes maurus (Sturm, 1827): (6569), 22.vi.2008, 1 spec.

Nebria brevicollis (Fabricius, 1792): (6569), 29.v.-11.vii.2008, PFT - locality 3, 1 spec.

Nebria livida (Linnaeus, 1758): (6569), 9.v.2008, 1 spec.; (6569), 17.viii.2008, 5 spec.

Notiophilus biguttatus (Fabricius, 1779): (6569), 19.x.2008, 1 spec.

Notiophilus pusillus G. R. Waterhouse, 1833: (6569), 17.viii.2008, 2 spec.

Omophron limbatum (Fabricius, 1777): (6569), 22.vi.2008, 2 spec.

Oodes helopioides (Fabricius, 1792): (6669), 9.v.2008, 1 spec.

Ophonus azureus (Fabricius, 1775): (6569), 11.vii.2008, on light, 5 spec.

Ophonus laticollis Mannerheim, 1825: (6569), 9.v.2008, 1 spec.; (6569), 29.v.-11.vii.2008, PFT - locality 4, 1 spec., P. Veselý det.; (6569), 11.vii.-17.viii.2008, PFT - locality 4, 2 spec., P. Veselý det.

Ophonus melletii (Heer, 1837): (6569), 29.v.-11.vii.2008, PFT - locality 3, 1 spec., P. Veselý det.; (6569), 11.vii.2008, on light, 2 spec., P. Veselý det.

Ophonus rufibarbis (Fabricius, 1792): (6569), 22.vi.2008, 1 spec., P. Veselý det.

Oxypselaphus obscurus (Herbst, 1784): (6569), 22.vi.2008, 2 spec.; (6569), 17.viii.2008, 1 spec.

Paranchus albipes (Fabricius, 1796): (6569), 29.v.2008, 2 spec.; (6569), 22.vi.2008, 1 spec.

Paratachys bistriatus (Duftschmid, 1812): (6569), 29.v.2008, 4 spec.; (6569), 14.vi.2008, 3 spec.; (6569), 22.vi.2008, 6 spec.; (6569), 11.vii.2008, on light, 2 spec.

Paratachys micros (Fischer von Waldheim, 1828): (6569), 29.v.2008, 3 spec.; (6569), 14.vi.2008, 1 spec.

Patrobus atrorufus (Stroem, 1768): (6569), 14.vi.2008, 1 spec.; (6569), 11.vii.-17.viii.2008, PFT - locality 1, 1 spec.; (6569), 17.viii.2008, 1 spec.

Perigona nigriceps (Dejean, 1831): (6569), 17.viii.2008, $1 \mathrm{spec}$.

Perileptus areolatus (Creutzer, 1799): (6569), 29.v.2008, 1 spec.; (6569), 22.vi.2008, 1 spec.

Philorhizus notatus (Stephens, 1827): (6569), 14.vi.2008, 1 spec., P. Veselý det.

Platynus assimilis (Paykull, 1790): (6569), 9.v.2008, 1 spec.; (6569), 29.v.-11.vii.2008, PFT - locality 1, 2 spec.; (6569), 29.v.-11.vii.2008, PFT - locality 2, 38 spec.; (6569), 29.v.-11.vii.2008, PFT - locality 3, 6 spec.; (6569), 29.v.-11.vii.2008, PFT - locality 4, 2 spec.; (6569), 11.vii.-17.viii.2008, PFT - locality 2, 1 spec.

Poecilus cupreus (Linnaeus, 1758): (6569), 11.vii.-17.viii.2008, PFT - locality 1, 1 spec.; (6569), 17.viii.2008, 3 spec. 
Pseudoophonus calceatus (Duftschmid, 1812): (6569), 11.vii.2008, on light, 1 spec.

Pseudoophonus griseus (Panzer, 1796): (6569), 11.vii.2008, on light, 1 spec.

Pseudoophonus rufipes (De Geer, 1774): (6569), 29.v.-11.vii.2008, PFT - locality 1, 2 spec.; (6569), 29.v.11.vii.2008, PFT - locality 2, 1 spec.; (6569), 29.v.-11.vii.2008, PFT - locality 3, 9 spec.; (6569), 11.vii.2008, on light, 3 spec.; (6569), 11.vii.-17.viii.2008, PFT - locality 1, 3 spec.; (6569), 11.vii.17.viii.2008, PFT - locality 2, 1 spec.; (6569), 11.vii.-17.viii.2008, PFT - locality 3, 14 spec.

Pterostichus anthracinus (Illiger, 1798): (6669), 9.v.2008, 2 spec.

Pterostichus melanarius (Illiger, 1798): (6569), 29.v.-11.vii.2008, PFT - locality 1, 3 spec.; (6569), 29.v.11.vii.2008, PFT - locality 2, 3 spec.; (6569), 29.v.-11.vii.2008, PFT - locality 3, 1 spec.; (6569), 11.vii.17.viii.2008, PFT - locality 1, 1 spec.; (6569), 11.vii.-17.viii.2008, PFT - locality 2, 10 spec.; (6569), 11.vii.-17.viii.2008, PFT - locality 3, 1 spec.; (6569), 11.vii.-17.viii.2008, PFT - locality 4, 1 spec.

Pterostichus niger (Schaller, 1783): (6569), 29.v.-11.vii.2008, PFT - locality 1, 1 spec.; (6569), 29.v.11.vii.2008, PFT - locality 3, 1 spec.; (6569), 29.v.-11.vii.2008, PFT - locality 4, 1 spec.; (6569), 11.vii.17.viii.2008, PFT - locality 4, 2 spec.

Pterostichus strenuus (Panzer, 1796): (6569), 9.v.2008, 1 spec.

Pterostichus vernalis (Panzer, 1796): (6569), 17.viii.2008, 1 spec.

Stenolophus discophorus Fischer von Waldheim, 1823: (6569), 11.vii.2008, on light, 1 spec.

Stenolophus mixtus (Herbst, 1784): (6569), 14.vi.2008, 1 spec.; (6569), 11.vii.2008, on light, 3 spec.

Stenolophus skrimshiranus Stephens, 1828: (6569), 11.vii.2008, on light, 1 spec.

Stenolophus teutonus (Schrank, 1781): (6569), 14.vi.2008, 1 spec.

Syntomus obscuroguttatus (Duftschmid, 1812): (6569), 19.x.2008, $1 \mathrm{spec}$.

Synuchus vivalis (Illiger, 1798): (6569), 11.vii.-17.viii.2008, PFT - locality 1, 1 spec.; (6569), 11.vii.17.viii.2008, PFT - locality 3, 1 spec.

Tachyta nana (Gyllenhal, 1810): (6569), 14.vi.2008, 1 spec.

Trechus pilisensis Csiki, 1918: (6569), 22.vi.2008, 9 spec.; (6569), 17.viii.2008, 1 spec.; (6569), 19.x.2008, 1 spec.

Trechus quadristriatus (Schrank, 1781): (6569), 22.vi.2008, 1 spec.; (6569), 17.viii.2008, 3 spec.

Trechus secalis secalis (Paykull, 1790): (6569), 17.viii.2008, 1 spec.

\section{HYDROPHILIDAE}

Anacaena lutescens (Stephens, 1829): (6569), 14.vi.2008, 1 spec.

Cercyon analis (Paykull, 1798): (6569), 9.v.2008, 2 spec.; (6569), 17.viii.2008, 4 spec.

Cercyon haemorrhoidalis (Fabricius, 1775): (6569), 22.vi.2008, in horse excrements, 1 spec.

Cercyon lateralis (Marsham, 1802): (6569), 22.vi.2008, 1 spec.; (6569), 17.viii.2008, 4 spec.; (6569), 19.x.2008, 1 spec.

Cercyon nigriceps (Marsham, 1802): (6569), 22.vi.2008, 1 spec.

Cercyon quisquilius (Linnaeus, 1760): (6569), 22.vi.2008, 1 spec.; (6569), 17.viii.2008, 1 spec.

Cercyon terminatus (Marsham, 1802): (6569), 22.vi.2008, 1 spec.

Coelostoma orbiculare (Fabricius, 1775): (6569), 11.vii.2008, 2 spec.

Cryptopleurum minutum (Fabricius, 1775): (6569), 22.vi.2008, 3 spec.

Cryptopleurum subtile Sharp, 1884: (6569), 17.viii.2008, 1 spec.

Enochrus quadripunctatus (Herbst, 1797): (6569), 11.vii.2008, on light, 1 spec.

Helochares obscurus (O. F. Müller, 1776): (6569), 11.vii.2008, on light, 2 spec.

Helophorus aequalis C.G.Thomson, 1868: (6569), 14.vi.2008, 1 spec.

Helophorus arvernicus Mulsant, 1846: (6569), 14.vi.2008, 1 spec.

Helophorus brevipalpis Bedel, 1881: (6569), 14.vi.2008, 1 spec.

Helophorus granularis (Linnaeus, 1760): (6569), 14.vi.2008, 2 spec.

Helophorus griseus Herbst, 1793: (6569), 9.v.2008, 3 spec.

Hydrobius fuscipes (Linnaeus, 1758): (6569), 14.vi.2008, 1 spec.; (6569), 11.vii.2008, on light, 1 spec.

Megasternum concinnum (Marsham, 1802): (6569), 17.viii.2008, 2 spec.; (6569), 19.x.2008, 2 spec.

Sphaeridium marginatum Fabricius, 1787: (6569), 22.vi.2008, in horse excrements, 19 spec.; (6569), 22.vi.2008, 1 spec.

Sphaeridium scarabaeoides (Linnaeus, 1758): (6569), 22.vi.2008, in horse excrements, 3 spec.

\section{HISTERIDAE}

Abraeus granulum Erichson, 1839: (6569), 14.vi.2008, together with Lasius fuliginosus, 1 spec.; (6569), 22.vi.2008, sifted from base of old tree, 1 spec. 
Abraeus perpusillus (Marsham, 1802): (6569), 14.vi.2008, 1 spec.; (6569), 22.vi.2008, sifted from base of old tree, 1 spec.; (6569), 19.x.2008, 12 spec.

Atholus bimaculatus (Linnaeus, 1758): (6569), 17.viii.2008, 1 spec.

Atholus duodecimstriatus duodecimstriatus (Schrank, 1781): (6569), 17.viii.2008, 2 spec.

Carcinops pumilio (Erichson, 1834): (6569), 17.viii.2008, 9 spec.

Gnathoncus nannetensis (Marseul, 1862): (6569), 14.vi.2008, 2 spec.

Hister unicolor Linnaeus, 1758: (6569), 22.vi.2008, in horse excrements, 1 spec.; (6569), 17.viii.2008, 1 spec.

Margarinotus carbonarius (Hoffmann, 1803): (6569), 17.viii.2008, 1 spec.; (6569), 11.vii.-17.viii.2008, PFT locality 4, 1 spec.

Margarinotus striola succicola (C. G. Thomson, 1862): (6569), 14.vi.2008, 2 spec.; (6569), 22.vi.2008, 2 spec.

Paromalus flavicornis (Herbst, 1792): (6569), 22.vi.2008, 6 spec.; (6569), 19.x.2008, 15 spec.

Plegaderus caesus (Herbst, 1792): (6569), 19.x.2008, 1 spec.

Saprinus semistriatus (Scriba, 1790): (6569), 14.vi.2008, on carrion, 2 spec.

\section{HYDRAENIDAE}

Hydraena riparia Kugelann, 1794: (6569), 14.vi.2008, 2 spec.

\section{PTILIIDAE}

Acrotrichis fascicularis (Herbst, 1793): (6569), 17.viii.2008, 1 spec.

Acrotrichis grandicollis (Mannerheim, 1844): (6569), 22.vi.2008, $6 \mathrm{spec}$.

Acrotrichis intermedia (Gillmeister, 1845): (6569), 22.vi.2008, sifted from base of old tree, 6 spec.; (6569), 19.x.2008, 1 spec.

Acrotrichis montandoni (Allibert, 1844): (6569), 17.viii.2008, 1 spec.; (6569), 19.x.2008, 8 spec.

Acrotrichis sericans (Heer, 1841): (6569), 22.vi.2008, 2 spec.

Ptenidium gressneri Erichson, 1845: (6569), 19.x.2008, 3 spec.

Ptenidium longicorne Fuss, 1868: (6569), 14.vi.2008, 19 spec.; (6569), 11.vii.2008, 3 spec.

Ptenidium pusillum (Gyllenhal, 1808): (6569), 17.viii.2008, 15 spec.

Ptenidium reitteri Flach, 1887: (6569), 29.v.2008, 1 spec.; (6569), 11.vii.2008, 1 spec.

Pteryx suturalis (Heer, 1841): (6569), 22.vi.2008, 1 spec.

\section{LEIODIDAE}

Anisotoma humeralis (Fabricius, 1792): (6569), 14.vi.2008, 1 spec.

Catops picipes (Fabricius, 1787): (6569), 14.vi.2008, 1 spec.; (6569), 22.vi.2008, sifted from base of old tree, 12 spec.

Fissocatops westi (Krogerus, 1931): (6569), 14.vi.2008, 1 spec.

Colenis immunda (Sturm, 1807): (6569), 14.vi.2008, 1 spec.; (6569), 11.vii.2008, 1 spec.

Sciodrepoides fumatus (Spence, 1815): (6569), 14.vi.2008, on carrion, 1 spec.; (6569), 11.vii.-17.viii.2008, PFT - locality 3, 1 spec.

Sciodrepoides watsoni (Spence, 1815): (6569), 14.vi.2008, on carrion, 2 spec.; (6569), 11.vii.-17.viii.2008, PFT - locality 3, 3 spec.; (6569), 17.viii.2008, 1 spec., O. Nakládal det.; (6569), 19.x.2008, 1 spec.

Agathidium seminulum (Linnaeus, 1758): (6569), 22.vi.2008, 1 spec.

Agathidium varians (Beck, 1817): (6569), 22.vi.2008, 2 spec.

Catops fuliginosus fuliginosus Erichson, 1837: (6569), 22.vi.2008, sifted from base of old tree, 29 spec., O. Nakládal det.; (6569), 22.vi.2008, sifted from base of old tree, 38 spec.; (6569), 19.x.2008, 2 spec.

Catops kirbyi kirbyi (Spence, 1815): (6569), 22.vi.2008, sifted from base of old tree, 8 spec., O. Nakládal det.; (6569), 22.vi.2008, sifted from base of old tree, 12 spec.; (6569), 11.vii.2008, 1 spec.;

Catops nigricans (Spence, 1815): (6569), 22.vi.2008, sifted from base of old tree, 3 spec.; (6569), 11.vii.2008, 1 spec.

Catops tristis tristis (Panzer, 1794): (6569), 22.vi.2008, sifted from base of old tree, 1 spec.

Catops neglectus Kraatz, 1852: (6569), 22.vi.2008, sifted from base of old tree, 4 spec.

Nemadus colonoides (Kraatz, 1851): (6569), 22.vi.2008, 1 spec.; (6569), 19.x.2008, 1 spec.

Anemadus strigosus strigosus (Kraatz, 1852): (6569), 11.vii.2008, 1 spec.

Nargus velox velox (Spence, 1815): (6569), 11.vii.2008, 3 spec.

Apocatops nigrita (Erichson, 1837): (6569), 11.vii.-17.viii.2008, PFT - locality 3, 2 spec.

Leiodes polita (Marsham, 1802): (6569), 11.vii.-17.viii.2008, PFT - locality 3., 1 spec.

Choleva spinipennis Reitter, 1890: (6569), 19.x.2008, burrow of small mammal, 1 spec., O. Nakládal det.

Ptomaphagus sericatus sericatus Chaudoir, 1845: (6569), 19.x.2008, 5 spec. 
Ptomaphagus varicornis (Rosenhauer, 1847): (6569), 19.x.2008, 1 spec.

\section{SCYDMAENIDAE}

Euconnus pragensis pragensis (Machulka, 1923): (6569), 14.vi.2008, 1 spec.; (6569), 22.vi.2008, 1 spec.

Euconnus pubicollis (P. W. J. Müller et Kunze, 1822): (6569), 14.vi.2008, 5 spec.; (6569), 19.x.2008, 1 spec. Neuraphes elongatulus (Müller \& Kunze, 1822): (6569), 14.vi.2008, 5 spec.; (6569), 22.vi.2008, 2 spec. Scydmaenus hellwigii (Herbst, 1792): (6569), 22.vi.2008, 2 spec.

Scydmaenus perrisi (Reitter, 1879): (6569), 22.vi.2008, 1 spec.

Scydmaenus tarsatus P. W. J. Müller et Kunze, 1822: (6569), 22.vi.2008, 1 spec.; (6569), 17.viii.2008, 2 spec. Scydmoraphes helvolus (Schaum, 1844): (6569), 14.vi.2008, 1 spec.; (6569), 11.vii.2008, 1 spec.; (6569), 19.x.2008, 2 spec.

Stenichnus collaris (P. W. J. Müller et Kunze, 1822): (6569), 11.vii.2008, 2 spec.

Stenichnus godarti (Latreille, 1806): (6569), 14.vi.2008, 3 spec.; (6569), 22.vi.2008, 3 spec.; (6569), 19.x.2008, 3 spec.

\section{SILPHIDAE}

Necrodes littoralis (Linnaeus, 1758): (6569), 11.vii.2008, 1 spec.

Nicrophorus humator (Gleditsch, 1767): (6569), 14.vi.2008, on carrion, 1 spec.

Nicrophorus interruptus Stephens, 1830: (6569), 11.vii.-17.viii.2008, PFT - locality 1, 7 spec.; (6569), 11.vii.17.viii.2008, PFT - locality 3, 61 spec.; (6569), 11.vii.-17.viii.2008, PFT - locality 3, 1 spec., J. Růžička det.; (6569), 11.vii.-17.viii.2008, PFT - locality 4, 1 spec.

Nicrophorus vespillo (Linnaeus, 1758): (6569), 29.v.-11.vii.2008, PFT - locality 1, 2 spec.; (6569), 29.v.11.vii.2008, PFT - locality 2, 3 spec.; (6569), 29.v.-11.vii.2008, PFT - locality 3, 2 spec.; (6569), 29.v.11.vii.2008, PFT - locality 4, 1 spec.; (6569), 14.vi.2008, on carrion, 2 spec.; (6569), 11.vii.-17.viii.2008, PFT - locality 1, 8 spec.; (6569), 11.vii.-17.viii.2008, PFT - locality 2, 12 spec.; (6569), 11.vii.17.viii.2008, PFT - locality 3, 71 spec.; (6569), 11.vii.-17.viii.2008, PFT - locality 3, 1 spec., J. Růžička det.; (6569), 11.vii.-17.viii.2008, PFT - locality 4, 4 spec.

Nicrophorus vespilloides Herbst, 1783: (6569), 14.vi.2008, on carrion, 1 spec., J. Růžička det.; (6569), 11.vii.-17.viii.2008, PFT - locality 1, 2 spec.; (6569), 11.vii.-17.viii.2008, PFT - locality 2, 8 spec.; (6569), 11.vii.-17.viii.2008, PFT - locality 3, 33 spec.

Oiceoptoma thoracicum (Linnaeus, 1758): (6569), 9.v.2008, 1 spec.; (6569), 29.v.-11.vii.2008, PFT - locality 4, 5 spec.; (6569), 11.vii.-17.viii.2008, PFT - locality 3, 2 spec.; (6669), 9.v.2008, 1 spec.

Phosphuga atrata (Linnaeus, 1758): (6569), 29.v.-11.vii.2008, PFT - locality 1, 13 spec.; (6569), 29.v.11.vii.2008, PFT - locality 3, 10 spec.; (6569), 29.v.-11.vii.2008, PFT - locality 4, 1 spec.; (6569), 11.vii.2008, 1 spec.; (6569), 11.vii.-17.viii.2008, PFT - locality 1, 16 spec.; (6569), 11.vii.-17.viii.2008, PFT - locality 2, 1 spec.; (6569), 11.vii.-17.viii.2008, PFT - locality 3, 13 spec.; (6569), 11.vii.17.viii.2008, PFT - locality 4, 7 spec.

Silpha carinata Herbst, 1783: (6569), 29.v.-11.vii.2008, PFT - locality 3, 7 spec.; (6569), 29.v.-11.vii.2008, PFT - locality 4, 2 spec.; (6569), 11.vii.-17.viii.2008, PFT - locality 3, 6 spec.; (6569), 11.vii.-17.viii.2008, PFT - locality 4, 5 spec.

Thanatophilus sinuatus (Fabricius, 1775): (6569), 22.vi.2008, on carrion, 1 spec.

\section{STAPHYLINIDAE}

Acrotona fungi (Gravenhorst, 1806): (6569), 19.x.2008, 3 spec.

Acrotona negligens (Mulsant et Rey, 1873): (6569), 9.v.2008, 1 spec.; (6569), 19.x.2008, 1 spec.

Acrotona orbata (Erichson, 1837): (6569), 19.x.2008, 1 spec.

Acrulia inflata (Gyllenhal, 1813): (6569), 19.x.2008, 1 spec.

Aleochara bipustulata (Linnaeus, 1760): (6569), 11.vii.2008, 1 spec.

Aleochara curtula (Goeze, 1777): (6569), 14.vi.2008, on carrion, 3 spec.; (6569), 11.vii.-17.viii.2008, PFT locality $1,1 \mathrm{spec}$.

Aleochara haematoptera Kraatz,1858: (6569), 29.v.2008, 3 spec.

Aleochara intricata Mannerheim, 1830: (6569), 22.vi.2008, 2 spec.

Aleochara lanuginosa Gravenhorst, 1802: (6569), 22.vi.2008, 1 spec.

Aleochara sparsa Heer, 1839: (6569), 14.vi.2008, 1 spec.; (6569), 19.x.2008, 2 spec.

Amischa bifoveolata (Mannerheim, 1830): (6569), 19.x.2008, 3 spec.

Anotylus insecatus (Gravenhorst, 1806): (6569), 9.v.2008, 1 spec. 
Anotylus rugosus (Fabricius, 1775): (6569), 22.vi.2008, 1 spec.; (6569), 11.vii.2008, on light, 8 spec.; (6569), 11.vii.2008, 1 spec.; (6569), 19.x.2008, 3 spec.

Anotylus sculpturatus (Gravenhorst, 1806): (6569), 14.vi.2008, 5 spec.; (6569), 22.vi.2008, 2 spec.; (6569), 17.viii.2008, 1 spec.; (6569), 11.vii.-17.viii.2008, PFT - locality 3, 1 spec.

Anotylus tetracarinatus (Block, 1799): (6569), 22.vi.2008, 8 spec.

Anthobium atrocephalum (Gyllenhal, 1827): (6569), 22.vi.2008, sifted from base of old tree, 7 spec.

Anthophagus angusticollis (Mannerheim, 1830): (6569), 14.vi.2008, 2 spec.

Anthophagus caraboides (Linnaeus, 1758): (6569), 14.vi.2008, 2 spec.

Aploderus caelatus (Gravenhorst, 1802): (6569), 22.vi.2008, 1 spec.

Astenus immaculatus Stephens, 1833: (6569), 19.x.2008, 1 spec.

Astenus pulchellus (Heer, 1839): (6569), 17.viii.2008, 2 spec.

Atheta crassicornis (Fabricius, 1793): (6569), 22.vi.2008, 1 spec.

Atheta hybrida Sharp, 1869: (6569), 22.vi.2008, sifted from base of old tree, 1 spec.

Atheta longicornis (Gravenhorst, 1802): (6569), 22.vi.2008, 2 spec.

Atheta ravilla (Erichson, 1839): (6569), 19.x.2008, 2 spec.

Atheta sodalis (Erichson, 1837): (6569), 9.v.2008, 1 spec.; (6569), 22.vi.2008, sifted from base of old tree, 1 spec.; (6569), 19.x.2008, 1 spec.

Atheta sordidula (Erichson, 1837): (6569), 19.x.2008, 2 spec.

Atheta triangulum (Kraatz, 1856): (6569), 9.v.2008, 1 spec.

Atheta trinotata (Kraatz, 1856): (6569), 22.vi.2008, sifted from base of old tree, 2 spec.

Autalia rivularis (Gravenhorst, 1802): (6569), 22.vi.2008, 1 spec.

Batrisodes delaporti (Aubé, 1833): (6569), 22.vi.2008, 3 spec.

Batrisus formicarius Aubé, 1833: (6569), 22.vi.2008, 2 spec.

Bisnius fimetarius (Gravenhorst, 1802): (6569), 14.vi.2008, 1 spec.; (6569), 22.vi.2008, 4 spec.; (6569), 17.viii.2008, 1 spec.; (6569), 19.x.2008, 4 spec.

Bledius crassicollis Lacordaire, 1835: (6569), 14.vi.2008, 1 spec.

Bledius cribricollis Heer, 1839: (6569), 11.vii.2008, 1 spec.

Bledius dissimilis Erichson, 1840: (6569), 14.vi.2008, 1 spec.

Bledius tricornis (Herbst, 1784): (6569), 11.vii.2008, on light, 1 spec.

Bolitobius cingulatus Mannerheim, 1830: (6669), 9.v.2008, 1 spec.

Bolitochara bella Märkel, 1845: (6569), 19.x.2008, 1 spec.

Bolitochara obliqua Erichson, 1837: (6569), 19.x.2008, 2 spec.

Brachygluta sinuata (Aubé, 1833): (6569), 22.vi.2008, 11 spec.; (6569), 19.x.2008, 6 spec.

Bythinus securiger securiger (Reichenbach, 1816): (6569), 22.vi.2008, 2 spec.

Carpelimus elongatulus (Erichson, 1839): (6569), 19.x.2008, 1 spec.

Carpelimus obesus (Kiesenwetter, 1844): (6569), 22.vi.2008, 1 spec.

Dinaraea angustula (Gyllenhal, 1810): (6569), 19.x.2008, burrow of small mammal, 1 spec.

Drusilla canaliculata (Fabricius, 1787): (6569), 11.vii.-17.viii.2008, PFT - locality 3, 1 spec.; (6569), 11.vii.17.viii.2008, PFT - locality 3, 1 spec.; (6569), 29.v.-11.vii.2008, PFT - locality 3, 1 spec.

Enalodroma hepatica (Erichson, 1839): (6569), 9.v.2008, 2 spec.

Erichsonius cinerascens (Gravenhorst, 1802): (6569), 14.vi.2008, 1 spec.

Euplectus karstenii (Reichenbach, 1816): (6569), 22.vi.2008, 3 spec.; (6569), 11.vii.2008, 1 spec.; (6569), 19.x.2008, 4 spec.

Euplectus nanus (Reichenbach, 1816): (6569), 22.vi.2008, 4 spec.

Euryusa pipitzi (Eppelsheim, 1887): (6569), 14.vi.2008, 2 spec., V. Assing det., O. Nakládal et P. Krásenský coll. (Nakládal \& Krásenský 2009).

Euryusa sinuata Erichson, 1837: (6569), 14.vi.2008, in nest of Lasius fuliginosus, 1 spec.

Gabrius osseticus (Kolenati, 1846): (6569), 22.vi.2008, sifted from base of old tree, 3 spec.; (6569), 11.vii.2008, 1 spec.; (6569), 17.viii.2008, 1 spec.; (6569), 19.x.2008, 3 spec.; (6569), 29.v.-11.vii.2008, PFT - locality 1, 1 spec.; (6569), 11.vii.-17.viii.2008, PFT - locality 3, 1 spec.

Gabrius splendidulus (Gravenhorst, 1802): (6569), 19.x.2008, 1 spec.

Gabrius suffragani Joy, 1913: (6569), 11.vii.2008, on light, 1 spec.

Geostiba circellaris (Gravenhorst, 1806): (6569), 22.vi.2008, sifted from base of old tree, 3 spec.

Gnypeta ripicola (Kiesenwetter, 1844): (6569), 29.v.2008, 1 spec.

Gyrohypnus fracticornis (O.F.Müller, 1776): (6569), 19.x.2008, 4 spec.

Gyrophaena manca Erichson, 1839: (6569), 17.viii.2008, 2 spec.

Habrocerus capillaricornis (Gravenhorst, 1806): (6569), 22.vi.2008, sifted from base of old tree, 2 spec.

Haploglossa gentilis (Märkel, 1845): (6569), 14.vi.2008, 1 spec.

Haploglossa marginalis (Gravenhorst, 1806): (6569), 11.vii.2008, 1 spec.

Haploglossa nidicola (Fairmaire, 1853): (6569), 19.x.2008, 1 spec. 
Haploglossa villosula (Stephens, 1832): (6569), 11.vii.2008, 1 spec.; (6569), 19.x.2008, 1 spec.

Heterothops dissimilis (Gravenhorst, 1802): (6569), 22.vi.2008, sifted from base of old tree, 4 spec.; (6569), 19.x.2008, 5 spec.

Heterothops praevius niger Kraatz, 1868: (6569), 19.x.2008, 1 spec.

Hypnogyra angularis (Ganglbauer, 1895): (6569), 9.v.2008, 1 spec.; (6569), 19.x.2008, 1 spec.

Ischnopoda leucopus (Marsham, 1802): (6569), 29.v.2008, $3 \mathrm{spec}$.

Ischnopoda umbratica (Erichson, 1837): (6569), 14.vi.2008, 1 spec.

Lathrobium brunnipes (Fabricius, 1793): (6569), 29.v.2008, 1 spec.

Lathrobium castaneipenne Kolenati, 1846: (6569), 17.viii.2008, 2 spec.

Lathrobium fulvipenne (Gravenhorst, 1806): (6569), 17.viii.2008, 1 spec.

Lithocharis ochracea (Gravenhorst, 1802): (6569), 17.viii.2008, 1 spec.

Medon fusculus (Mannerheim, 1830): (6569), 22.vi.2008, sifted from base of old tree, 1 spec.

Megarthrus depressus (Paykull, 1789): (6569), 9.v.2008, 2 spec.; (6569), 17.viii.2008, 1 spec.

Meotica filiformis (Motschulsky, 1860): (6569), 9.v.2008, 1 spec.

Micropeplus marietti Jacquelin du Val, 1857: (6569), 9.v.2008, 2 spec.

Nehemitropia lividipennis (Mannerheim, 1830): (6569), 22.vi.2008, 2 spec.

Neobisnius prolixus (Erichson, 1840): (6569), 22.vi.2008, 1 spec.

Neobisnius villosulus (Stephens, 1833): (6569), 29.v.2008, 3 spec.; (6569), 14.vi.2008, 1 spec.

Ocypus nitens nitens (Schrank, 1781): (6569), 29.v.2008, 1 spec.; (6569), 29.v.-11.vii.2008, PFT - locality 3, 2 spec.

Oligota pusillima (Gravenhorst, 1806): (6569), 9.v.2008, 2 spec.; (6569), 22.vi.2008, sifted from base of old tree, 1 spec.

Omalium caesum Gravenhorst, 1806: (6569), 22.vi.2008, sifted from base of old tree, 16 spec.

Omalium rivulare (Paykull, 1789): (6569), 17.viii.2008, 1 spec.; (6569), 19.x.2008, 7 spec.

Ontholestes haroldi (Eppelsheim, 1884): (6569), 9.v.2008, 1 spec.; (6569), 11.vii.-17.viii.2008, PFT - locality 3,3 spec.

Ontholestes murinus (Linnaeus, 1758): (6569), 22.vi.2008, on carrion, 1 spec.; (6569), 17.viii.2008, 1 spec.

Othius punctulatus (Goeze, 1777): (6569), 29.v.-11.vii.2008, PFT - locality 3, 1 spec.

Oxypoda opaca (Gravenhorst, 1802): (6569), 14.vi.2008, in nest of Lasius fuliginosus, 1 spec.; (6569), 19.x.2008, 1 spec.

Oxypoda vittata Märkel, 1842: (6569), 22.vi.2008, sifted from base of old tree, 3 spec.

Oxytelus sculptus Gravenhorst, 1806: (6569), 22.vi.2008, 1 spec.

Paederidus ruficollis (Fabricius, 1777): (6569), 29.v.2008, 2 spec.; (6569), 14.vi.2008, 2 spec.

Paederus limnophilus Erichson, 1840: (6569), 29.v.2008, 5 spec.; (6569), 14.vi.2008, 2 spec.; (6669), 9.v.2008, 3 spec.

Paederus littoralis Gravenhorst, 1802: (6569), 14.vi.2008, 2 spec.; (6569), 19.x.2008, burrow of small mammal, 1 spec.

Paederus schoenherri Czwalina, 1889: (6569), 9.v.2008, 1 spec.; (6569), 14.vi.2008, 1 spec.

Phacophallus parumpunctatus (Gyllenhal, 1827): (6569), 17.viii.2008, 1 spec.

Philonthus addendus Sharp in Crotch et Sharp, 1867: (6569), 19.x.2008, 1 spec.; (6569), 11.vii.-17.viii.2008, PFT - locality 4, 1 spec.; (6569), 11.vii.-17.viii.2008, PFT - locality 3, 1 spec.

Philonthus atratus (Gravenhorst, 1802): (6569), 29.v.2008, 2 spec.; (6669), 9.v.2008, 1 spec.

Philonthus cognatus Stephens, 1832: (6569), 11.vii.2008, 1 spec.; (6669), 9.v.2008, 1 spec.

Philonthus decorus (Gravenhorst, 1802): (6569), 11.vii.-17.viii.2008, PFT - locality 1, 2 spec.; (6569), 11.vii.17.viii.2008, PFT - locality 2, 7 spec.; (6569), 29.v.-11.vii.2008, PFT - locality 1, 5 spec.; (6569), 29.v.11.vii.2008, PFT - locality 2, 2 spec.; (6569), 29.v.-11.vii.2008, PFT - locality 3, 7 spec.; (6569), 29.v.11.vii.2008, PFT - locality 4, 2 spec.

Philonthus fumarius (Gravenhorst, 1806): (6569), 14.vi.2008, 1 spec.

Philonthus longicornis Stephens, 1832: (6569), 22.vi.2008, 2 spec.

Philonthus micans (Gravenhorst, 1802): (6569), 29.v.2008, 1 spec.; (6569), 14.vi.2008, 1 spec.

Philonthus pseudovarians A. Strand, 1941: (6569), 11.vii.-17.viii.2008, PFT - locality 3, 1 spec.

Philonthus quisquiliarius (Gyllenhal, 1810): (6569), 29.v.2008, 1 spec.; (6569), 11.vii.2008, on light, 3 spec.; (6669), 9.v.2008, 1 spec.

Philonthus rectangulus Sharp, 1874: (6569), 22.vi.2008, 2 spec.

Philonthus rotundicollis (Ménétriés, 1832): (6569), 14.vi.2008, $1 \mathrm{spec}$.

Philonthus rubripennis Stephens, 1832: (6569), 29.v.2008, 4 spec.; (6569), 14.vi.2008, 7 spec.; (6569), 22.vi.2008, 1 spec.

Philonthus sanguinolentus (Gravenhorst, 1802): (6569), 22.vi.2008, $1 \mathrm{spec}$.

Philonthus succicola C.G.Thomson, 1860: (6569), 19.x.2008, 1 spec.; (6569), 11.vii.-17.viii.2008, PFT locality 4, 1 spec.; (6569), 11.vii.-17.viii.2008, PFT - locality 3, 1 spec. 
Philonthus tenuicornis Rey, 1853: (6569), 14.vi.2008, 1 spec.; (6569), 9.v.2008, 1 spec.; (6569), 19.x.2008, 1 spec.; (6569), 11.vii.-17.viii.2008, PFT - locality 3, 1 spec.

Philonthus varians (Paykull, 1789): (6569), 22.vi.2008, 1 spec.; (6569), 17.viii.2008, 2 spec.

Phloeocharis subtilissima Mannerheim, 1830: (6569), 22.vi.2008, sifted from base of old tree, 1 spec.; (6569), 11.vii.2008, 1 spec.

Phloeonomus punctipennis C.G.Thomson, 1867: (6569), 19.x.2008, 1 spec.

Phyllodrepa floralis (Paykull, 1789): (6569), 14.vi.2008, in nest of Lasius fuliginosus, 2 spec.; (6569), 19.x.2008, 1 spec.

Phyllodrepa ioptera (Stephens, 1834): (6569), 22.vi.2008, sifted from base of old tree, 8 spec.

Phyllodrepa melanocephala (Fabricius, 1787): (6569), 22.vi.2008, sifted from base of old tree, 5 spec.; (6569), 11.vii.2008, 1 spec.

Platystethus arenarius (Geoffroy, 1785): (6569), 22.vi.2008, 3 spec.

Platystethus cornutus (Gravenhorst, 1802): (6569), 22.vi.2008, 3 spec.

Platystethus nitens (C.R.Sahlberg, 1832): (6569), 22.vi.2008, 1 spec.; (6569), 17.viii.2008, 1 spec.

Plectophloeus nubigena nubigena (Reitter, 1877): (6569), 19.x.2008, 1 spec.

Quedius cruentus (Olivier, 1795): (6569), 14.vi.2008, 3 spec.

Quedius fuliginosus (Gravenhorst, 1802): (6569), 29.v.-11.vii.2008, PFT - locality 3, 2 spec.

Quedius limbatus Heer, 1839: (6569), 22.vi.2008, sifted from base of old tree, 2 spec.

Quedius longicornis Kraatz, 1857: (6569), 19.x.2008, burrow of small mammal, 1 spec.

Quedius umbrinus Erichson, 1839: (6569), 14.vi.2008, 1 spec.

Rugilus orbiculatus (Paykull, 1789): (6569), 9.v.2008, 1 spec.; (6569), 17.viii.2008, 1 spec.

Rugilus rufipes Germar, 1836: (6569), 14.vi.2008, in nest of Lasius fuliginosus, 2 spec.; (6569), 22.vi.2008, sifted from base of old tree, 2 spec.; (6569), 19.x.2008, 1 spec.

Scaphisoma agaricinum (Linnaeus, 1758): (6569), 17.viii.2008, $1 \mathrm{spec}$.

Sepedophilus marshami (Stephens, 1832): (6569), 22.vi.2008, sifted from base of old tree, 1 spec.

Sepedophilus testaceus (Fabricius, 1793): (6569), 14.vi.2008, in nest of Lasius fuliginosus, 1 spec.; (6569), 19.x.2008, 2 spec.

Siagonium quadricorne Kirby et Spence, 1815: (6569), 11.vii.2008, 3 spec.

Stenus ater Mannerheim, 1830: (6569), 9.v.2008, 1 spec.

Stenus boops Ljungh, 1804: (6569), 29.v.2008, 1 spec.; (6569), 14.vi.2008, 1 spec.

Stenus cicindeloides (Schaller, 1783): (6569), 29.v.2008, 1 spec.

Stenus clavicornis (Scopoli, 1763): (6569), 9.v.2008, 1 spec.

Stenus comma Leconte, 1863: (6569), 9.v.2008, 2 spec.; (6569), 29.v.2008, 4 spec.; (6569), 14.vi.2008, 2 spec.; (6569), 22.vi.2008, 2 spec.

Stenus humilis Erichson, 1839: (6569), 22.vi.2008, 1 spec.; (6569), 19.x.2008, 1 spec.

Stenus ludyi Fauvel, 1886: (6569), 22.vi.2008, sifted from base of old tree, 5 spec.

Stenus similis (Herbst, 1784): (6569), 29.v.2008, 1 spec.; (6569), 22.vi.2008, 1 spec.

Sunius melanocephalus (Fabricius, 1793): (6569), 19.x.2008, 1 spec.

Syntomium aeneum (P. Müller, 1821): (6569), 14.vi.2008, in nest of Lasius fuliginosus, 1 spec.

Tachinus fimetarius Gravenhorst, 1802: (6569), 22.vi.2008, 1 spec.

Tachinus rufipes (Linnaeus, 1758): (6569), 29.v.-11.vii.2008, PFT - locality 4, 7 spec.; (6569), 14.vi.2008, 1 spec.; (6569), 17.viii.2008, 1 spec.

Tachinus subterraneus (Linnaeus, 1758): (6569), 9.v.2008, 1 spec.

Tachyporus abdominalis (Fabricius, 1781): (6569), 17.viii.2008, 1 spec.

Tachyporus hypnorum (Fabricius, 1775): (6569), 19.x.2008, 2 spec.

Tachyporus nitidulus (Fabricius, 1781): (6569), 17.viii.2008, 1 spec.

Tachyporus obtusus (Linnaeus, 1767): (6569), 19.x.2008, 1 spec.

Tachyporus solutus Erichson, 1839: (6569), 9.v.2008, 1 spec.; (6569), 14.vi.2008, 1 spec.

Tachyusa constricta Erichson, 1837: (6569), 14.vi.2008, 5 spec.

Tasgius melanarius (Heer, 1839): (6569), 11.vii.-17.viii.2008, PFT - locality 1, 1 spec.; (6569), 29.v.11.vii.2008, PFT - locality 1, 2 spec.

Tasgius morsitans (Rossi, 1790): (6569), 11.vii.-17.viii.2008, PFT - locality 1, 1 spec.

Tasgius winkleri (Bernhauer, 1906): (6569), 17.viii.2008, 1 spec.

Thamiaraea cinnamomea (Gravenhorst, 1802): (6569), 14.vi.2008, 4 spec.

Thoracophorus corticinus Motschulsky, 1837: (6569), 22.vi.2008, sifted from base of old tree, 1 spec.

Tinotus morion (Gravenhorst, 1802): (6569), 22.vi.2008, 1 spec.

Trimium brevicorne (Reichenbach, 1816): (6569), 22.vi.2008, 1 spec.; (6569), 11.vii.2008, 2 spec.

Trissemus antennatus serricornis (Schmidt-Goebel, 1838): (6569), 14.vi.2008, 1 spec.

Xantholinus tricolor (Fabricius, 1787): (6569), 17.viii.2008, 1 spec.

Xylodromus affinis (Gerhardt, 1877): (6569), 11.vii.2008, 1 spec. 
Xylodromus concinnus (Marsham, 1802): (6569), 11.vii.2008, 1 spec.

Xylostiba bosnica (Bernhauer, 1902): (6569), 19.x.2008, 1 spec.

Zyras cognatus (Märkel, 1842): (6569), 22.vi.2008, 1 spec.; (6569), 19.x.2008, 1 spec.

Zyras funestus (Gravenhorst, 1806): (6569), 22.vi.2008, sifted from base of old tree, 1 spec.; (6569), 19.x.2008, 1 spec.

Zyras humeralis (Gravenhorst, 1802): (6569), 14.vi.2008, in nest of Lasius fuliginosus, 1 spec.

Zyras laticollis (Märkel, 1845): (6569), 22.vi.2008, sifted from base of old tree, 1 spec.; (6569), 19.x.2008, 2 spec.

Zyras limbatus (Paykull, 1789): (6569), 29.v.-11.vii.2008, PFT - locality 3, 1 spec.

Zyras lugens (Gravenhorst, 1802): (6569), 14.vi.2008, in nest of Lasius fuliginosus, 3 spec.

\section{LUCANIDAE}

Dorcus parallelipipedus (Linnaeus, 1758): (6569), 14.vi.2008, 1 spec.; (6569), 11.vii.-17.viii.2008, PFT locality 2, 1 spec.

\section{GEOTRUPIDAE}

Anoplotrupes stercorosus (Scriba, 1791): (6569), 11.vii.-17.viii.2008, PFT - locality 2, 1 spec.

\section{SCARABAEIDAE}

Aphodius fimetarius (Linnaeus, 1758): (6569), 22.vi.2008, in horse excrements, 1 spec.

Aphodius corvinus Erichson, 1848: (6569), 14.vi.2008, 1 spec.

Aphodius depressus (Kugelann, 1792): (6569), 11.vii.-17.viii.2008, PFT - locality 1, 1 spec.; (6569), 17.viii.2008, 3 spec.

Aphodius maculatus Sturm, 1800: (6569), 17.viii.2008, 4 spec.

Aphodius prodromus (Brahm, 1790): (6569), 29.v.-11.vii.2008, PFT - locality 3, 1 spec.; (6569), 22.vi.2008, in horse excrements, 1 spec.; (6569), 19.x.2008, 3 spec.; (6569), 19.x.2008, 1 spec.

Aphodius pusillus (Herbst, 1789): (6569), 22.vi.2008, in horse excrements, 1 spec.

Aphodius reyi Reitter, 1892: (6569), 19.x.2008, 1 spec.

Aphodius sticticus (Panzer, 1798): (6569), 14.vi.2008, 3 spec.; (6569), 22.vi.2008, in horse excrements, 1 spec.

Cetonia aurata (Linnaeus, 1761): (6569), 22.vi.2008, 1 spec., O. Nakládal det.

Melolontha melolontha (Linnaeus, 1758): (6569), 9.v.2008, 2 spec., O. Nakládal det.

Onthophagus coenobita (Herbst, 1783): (6569), 22.vi.2008, in horse excrements, 17 spec.

Onthophagus fracticornis (Preyssler, 1790): (6569), 22.vi.2008, in horse excrements, 4 spec.

Onthophagus joannae Goljan, 1953: (6569), 22.vi.2008, in horse excrements, 8 spec.; (6569), 17.viii.2008, 1 spec.

Onthophagus ovatus (Linnaeus, 1758): (6569), 22.vi.2008, in horse excrements, 9 spec.; (6569), 11.vii.17.viii.2008, PFT - locality 3, 4 spec.

Onthophagus ruficapillus Brullé, 1832: (6569), 17.viii.2008, 1 spec.

Oxyomus sylvestris (Scopoli, 1763): (6569), 22.vi.2008, 1 spec., O. Nakládal det.; (6569), 17.viii.2008, 2 spec., O. Nakládal det.

Oxythyrea funesta (Poda, 1761): (6569), 9.v.2008, 2 spec., O. Nakládal det.; (6569), 29.v.2008, 1 spec., O. Nakládal det.

Pleurophorus caesus (Creutzer, 1796): (6569), 11.vii.2008, 1 spec., O. Nakládal det.

Protaetia fieberi (Kraatz, 1880): (6569), 22.vi.2008, 1 spec.

Valgus hemipterus (Linnaeus, 1758): (6569), 9.v.2008, 2 spec., O. Nakládal det.; (6569), 22.vi.2008, 1 spec., O. Nakládal det.; (6669), 9.v.2008, 2 spec., O. Nakládal det.

\section{BUPRESTIDAE}

Agrilus angustulus angustulus (Illiger, 1803): (6569), 9.v.2008, 1 spec.; (6569), 14.vi.2008, 2 spec.; (6569), 22.vi.2008, 6 spec.

Agrilus convexicollis L.Redtenbacher, 1849: (6569), 14.vi.2008, 1 spec.; (6569), 22.vi.2008, 1 spec.; (6569), 11.vii.2008, 1 spec.

Agrilus laticornis (Illiger, 1803): (6569), 29.v.2008, 1 spec.; (6569), 14.vi.2008, 2 spec.; (6569), 22.vi.2008, 1 spec.; (6569), 11.vii.2008, 1 spec.

Anthaxia nitidula (Linnaeus, 1758): (6569), 9.v.2008, 1 spec.; (6569), 29.v.2008, 2 spec.; (6669), 9.v.2008, 1 spec. 
Anthaxia podolica podolica (Mannerheim, 1837): (6569), 14.vi.2008, 9 spec.; (6569), 22.vi.2008, 1 spec.; (6569), 11.vii.2008, 2 spec.; (6569), 17.viii.2008, 3 spec.

Coraebus elatus (Fabricius, 1787): (6569), 11.vii.2008, 1 spec., O. Nakládal det.

Trachys minuta (Linnaeus, 1758): (6569), 14.vi.2008, 5 spec., O. Nakládal det.

\section{CEROPHYTIDAE}

Cerophytum elateroides (Latreille, 1809): (6569), 9.v.2008, 1 spec.; (6569), 11.vii.2008, dead in spider web on old maple (Acer sp.), 1 spec.

\section{EUCNEMIDAE}

Hylis foveicollis (Thomson, 1874): (6569), 11.vii.2008, 2 spec.

\section{THROSCIDAE}

Trixagus dermestoides (Linnaeus, 1767): (6569), 29.v.2008, 1 spec.; (6569), 14.vi.2008, 1 spec.

Trixagus elateroides (Heer, 1841): (6569), 29.v.2008, 2 spec.; (6569), 14.vi.2008, 1 spec.

\section{ELATERIDAE}

Adrastus rachifer (Geoffroy, 1785): (6569), 22.vi.2008, 2 spec.; (6569), 11.vii.2008, 18 spec.; (6669), 22.vi.2008, 4 spec.

Agriotes acuminatus (Stephens, 1830): (6569), 9.v.2008, 3 spec.; (6569), 29.v.2008, 1 spec.

Agriotes obscurus (Linnaeus, 1758): (6569), 29.v.2008, 1 spec.; (6569), 14.vi.2008, 3 spec.

Agriotes sputator (Linnaeus, 1758): (6569), 9.v.2008, 2 spec.; (6569), 29.v.2008, 3 spec.; (6569), 22.vi.2008, 1 spec.; (6569), 11.vii.2008, 1 spec.

Agriotes ustulatus (Schaller, 1783): (6569), 11.vii.2008, 4 spec.

Agrypnus murinus (Linnaeus, 1758): (6569), 9.v.2008, 1 spec., O. Nakládal det.; (6569), 29.v.2008, 3 spec., O. Nakládal det.; (6569), 29.v.-11.vii.2008, PFT - locality 3, 3 spec., O. Nakládal det.; (6569), 11.vii.17.viii.2008, PFT - locality 3, 2 spec., O. Nakládal det.

Ampedus pomorum (Herbst, 1784): (6569), 9.v.2008, 1 spec.

Athous bicolor (Goeze, 1777): (6569), 14.vi.2008, 1 spec.; (6569), 22.vi.2008, 4 spec.; (6569), 11.vii.2008, 4 spec.

Athous haemorrhoidalis (Fabricius, 1801): (6569), 9.v.2008, 2 spec.; (6569), 29.v.2008, 7 spec.; (6569), 22.vi.2008, 1 spec.

Athous subfuscus (O.F.Müller, 1764): (6569), 9.v.2008, 2 spec.; (6569), 29.v.2008, 1 spec.

Athous vittatus (Fabricius, 1792): (6569), 9.v.2008, 2 spec.; (6569), 29.v.2008, 1 spec.; (6569), 14.vi.2008, 2 spec.

Cidnopus pilosus (Leske, 1785): (6569), 9.v.2008, 1 spec.; (6569), 29.v.2008, 1 spec.

Dalopius marginatus (Linnaeus, 1758): (6569), 9.v.2008, 3 spec.; (6569), 29.v.2008, 1 spec.

Denticollis linearis (Linnaeus, 1758): (6569), 9.v.2008, 1 spec.; (6569), 29.v.2008, 2 spec., O. Nakládal det.

Hemicrepidius hirtus (Herbst, 1784): (6569), 22.vi.2008, 3 spec.

Hemicrepidius niger (Linnaeus, 1758): (6569), 14.vi.2008, 4 spec.

Limonius poneli Leseigneur et Mertlik, 2007: (6569), 29.v.2008, 2 spec.

Melanotus villosus (Geoffroy, 1785): (6569), 9.v.2008, 2 spec.

Negastrius pulchellus (Linnaeus, 1761): (6569), 29.v.2008, 3 spec.

Negastrius sabulicola (Boheman, 1854): (6569), 29.v.2008, 8 spec.

Procraerus tibialis (Lacordaire, 1835): (6569), 19.x.2008, 1 spec.

Stenagostus rhombeus (Olivier, 1790): (6569), 11.vii.2008, on light, 1 spec., O. Nakládal det.

Synaptus filiformis (Fabricius, 1781): (6569), 9.v.2008, 3 spec.; (6569), 29.v.2008, 3 spec.; (6569), 14.vi.2008, 2 spec.; (6569), 11.vii.2008, 1 spec.

Zorochros dermestoides (Herbst, 1806): (6569), 29.v.2008, 8 spec.; (6569), 14.vi.2008, 3 spec.

\section{DRILIDAE}

Drilus concolor Ahrens, 1812: (6569), 14.vi.2008, 1 spec.; (6569), 22.vi.2008, 2 spec. 


\section{LYCIDAE}

Erotides cosnardi (Chevrolat, 1831): (6569), 9.v.2008, 1 spec., V. Švihla det.

Lygistopterus sanguineus (Linnaeus, 1758): (6569), 11.vii.2008, 1 spec.

\section{LAMPYRIDAE}

Phosphaenus hemipterus (Geoffroy, 1762): (6569), 22.vi.2008, 1 spec.

\section{CANTHARIDAE}

Cantharis fusca Linnaeus, 1758: (6569), 29.v.2008, 3 spec.

Cantharis lateralis Linnaeus, 1758: (6569), 22.vi.2008, 1 spec.; (6569), 11.vii.2008, 1 spec.; (6669), 22.vi.2008, 4 spec.

Cantharis livida Linnaeus, 1758: (6569), 29.v.2008, 19 spec.; (6669), 9.v.2008, 1 spec.

Cantharis nigra (De Geer, 1774): (6569), 11.vii.2008, on light, 1 spec.

Cantharis nigricans O.F.Müller, 1776: (6569), 9.v.2008, 2 spec.; (6569), 29.v.2008, 7 spec.; (6569), 14.vi.2008, 1 spec.; (6669), 9.v.2008, 1 spec.

Cantharis pellucida Fabricius, 1792: (6569), 9.v.2008, 4 spec.; (6569), 29.v.2008, 3 spec.; (6669), 9.v.2008, 2 spec.

Cantharis rufa Linnaeus, 1758: (6569), 29.v.2008, 4 spec.; (6569), 22.vi.2008, 1 spec.; (6569), 11.vii.2008, 1 spec.

Cantharis rustica Fallen, 1807: (6669), 9.v.2008, 1 spec.

Malthinus flaveolus (Herbst, 1786): (6569), 14.vi.2008, 1 spec.; (6569), 22.vi.2008, 2 spec.

Malthodes europaeus Wittmer, 1970: (6569), 9.v.2008, 1 spec.

Malthodes lobatus Kiesenwetter, 1852: (6569), 22.vi.2008, 1 spec.

Malthodes marginatus (Latreille, 1806): (6569), 9.v.2008, 3 spec.; (6569), 29.v.2008, 1 spec.

Metacantharis discoidea (Ahrens, 1812): (6569), 9.v.2008, 1 spec.

Rhagonycha fulva (Scopoli, 1763): (6569), 22.vi.2008, 1 spec.; (6569), 11.vii.2008, 2 spec.; (6569), 11.vii.17.viii.2008, PFT - locality 3,1 spec., O. Nakládal det.

Rhagonycha gallica Pic, 1923: (6569), 9.v.2008, 1 spec.; (6569), 29.v.2008, 1 spec.; (6569), 14.vi.2008, 1 spec.

Rhagonycha lutea (O.F.Müller, 1764): (6569), 14.vi.2008, 3 spec.; (6569), 22.vi.2008, 1 spec.

Rhagonycha nigriventris Motschulsky, 1860: (6669), 9.v.2008, 1 spec.

Silis nitidula (Fabricius, 1792): (6569), 22.vi.2008, 4 spec.

\section{Dicussion}

During the all survey 1 species belonging to the NATURA 2000 was recorded, next 5 species are protected by law in the Czech Republic, 4 species are critically endangered according to Red list of theatened species in the Czech Republic (abbreviated as RL) (Farkač et al. 2005), 15 endangered (RL), 15 near theatened (RL), 32 vulnerable (RL). In following text, only species with faunistic data presinting in this paper are listed.

Species protected by law in the Czech Republic: Brachinus explodens, Carabus scheidleri, Carabus ullrichii and Cicindela arenaria viennensis (all Carabidae) and Oxythyrea funesta (Scarabaeidae).

Vulnerable (according RL): Acupalpus luteatus, Agonum lugens, Bembidion modestum, Chlaenius tristis, Dyschirius nitidus, Stenolophus discophorus (Carabidae), Cercyon nigriceps (Hydrophilidae), Abraeus granulum (Histeridae), Choleva spinipennis (Leiodidae), Bledius dissimilis, Euryusa sinuata, Haploglossa gentilis, Haploglossa marginalis, Haploglossa nidicola, Heterothops praevius niger, Ontholestes haroldi, Philonthus pseudovarians, Tasgius winkleri, Thamiaraea cinnamomea, Trissemus antennatus serricornis (Staphylinidae), Agrilus convexicollis (Buprestidae), Drilus concolor (Drilidae), Phosphaenus hemipterus (Lampyridae) and Malthodes lobatus (Cantharidae).

Near theatened (according RL): Bembidion fluviatile, Dyschirius intermedius, Nebria livida, Perileptus areolatus (Carabidae), Nemadus colonoides (Leiodidae), Aphodius reyi, Onthophagus ruficapillus, Pleurophorus caesus (Scarabaeidae), Coraebus elatus (Buprestidae) and Adrastus rachifer (Elateridae). 
Endangered (according RL): Bembidion testaceum (Carabidae), Bledius tricornis, Stenus ludyi, Thoracophorus corticinus, Xantholinus tricolor, Xylostiba bosnica (Staphylinidae), Protaetia fieberi (Scarabaeidae), Hylis foveicollis (Eucnemidae) and Stenagostus rhombeus (Elateridae).

Critically endangered (according RL): Cerophytum elateroides (Cerophytidae) is known from the Czech Republic on base of old records of Prague environs (Fleischer 1927-1930). Recently discovered only from Hukvaldy game park in north Moravia (Vávra 1995). The species is associated with old moulder wood of deciduous trees (e.g. lindens, poplars) in which larvae develps (Burakowski 1991). Adults are usually discovered under the bark or inside of the wood, where are hidden during the day (Vávra 2005). They are active during the twilight and the night (Burakowski et al. 1985).

Negastrius pulchellus and N. sabulicola (Elateridae) are species developing in sandy soil near rivers and lakes. Larvae are probably polyphagous. Overwintering adults emerg from spring to summer among gravel and greensward vegetation. It is local species spread through the all regian of the Czech Republic (Laibner 2000). Both species are very common in study area.

\section{Conclusions}

Inventory survey of beetles (Coleoptera) was made in Zástudánčí NNR. NNR is sited in Central Moravia by the southeastern from Tovačov town. 3585 specimens belonging to 857 species from 64 families were recorded. Faunistic data of 23 of them (Haliplidae, Dytiscidae, Carabidae, Hydrophilidae, Histeridae, Hydraenidae, Ptiliidae, Leiodidae, Scydmaenidae, Silphidae, Staphylinidae, Lucanidae, Geotrupidae, Scarabaeidae, Buprestidae, Cerophytidae, Eucnemidae, Throscidae, Elateridae, Drilidae, Lycidae, Lampyridae, Cantharidae) are presented. Presenting part of data represent 2191 specimens belonging to 456 species.

One recorded species belong to the NATURA 2000, 4 ones are endangered according to notice of Ministry of the Environment of the Czech Republic no. 395/1992 code fo law. Four critically endangered, 15 endangered, 15 near threatened and 32 vulnerable species according Red list of threatened species in the Czech Republic (Farkač et al. 2005) were also recorded.

Majority of these interesting species are associate to old treestands or only to single old trees (very old succession stage) or conversely on very early succession stages like sandy and gravely riparial zone along the Morava river are. Managenet of nature preservation should be evolved from this findings. Whereas, riparial zone is not endangered (in case conserving nutural river dynamics), old trees particularly on dam (beyond the northwestern boundary of Zástudánčí National Nature Reserve - see the Fig. 1) can be endangered by direct liquidation in the future. Just this part is very valuable in nature preservaton point of view.

Acknowledgement: Thanks are due to the employee of the administration of the Litovelské Pomoraví Protected Landscape Area, Václav Polášek, for his kind help with collection permits and during the fieldwork. I am obliged to all those who made me identification of some families. This paper was written in the framework of the research project NAZV 81136.

\section{References}

Burakowski B. (1991): Klucze do oznaczania owadów Polski. Cz. 19, Zesz. 35-37. Chrząszcze - Coleoptera (Cerophytidae, Eucnemidae, Throscidae, Lissomidae). 91 pp., PTE, Wrocław.

Burakowski B., Mroczkowski M. \& Stefańska J. (1985): Katalog fauny Polski. Catalogus faunae Poloniae. Part 23, vol. 10., Chrząszcze. Coleoptera. Buprestoidea, Elateroidea i Cantharoidea. 401 pp., Panstwowe wydawnictwo naukowe, Warszawa.

Čelechovská J. (in litt.): Denní motýli (Lepidoptera: Rhopalocera) v okolí Tovačova. (Diplomová práce). [Daily butterfly (Lepidoptera: Rhopalocera) of Central Moravia region] (Diploma thesis)]. Faculty of Science, Palacký University Olomouc, 2001, 73 pp., Olomouc.

Čelechovský A. (2001b): Motýli (Macrolepidoptera) NPR Zástudánčí u Tovačova. Macrolepidoptera of the Zástudánčí Nature Reserve near Tovačov. Př́rodověd. Stud. Muz. (Prostějov), 4: 103-114. 
- (in litt.): Píd'alkovití (Geometridae, Lepidoptera) okolí Tovačova. (Diplomová práce). [Geometridae (Lepidoptera) of Tovačov town environs] (Unpublished diploma thesis). Faculty of Science, Palacký University Olomouc, 1993, 112 pp., Olomouc.

- (in litt.): Denní motýli (Lepidoptera: Rhopalocera) na území střední Moravy. (Disertační práce). [Daily butterfly (Lepidoptera: Rhopalocera) of Central Moravia region] (Ph.D. thesis). Faculty of Science, Palacký University Olomouc, 2001a, 130 pp., Olomouc.

Farkač J., Král D. \& Šk orpík M. (2005): Červený seznam ohrožených druhů České Republiky - Bezobratlí. (Red list of threatened species in the Czech Republic - Invertebrates). 758 pp., AOPK, Praha.

Fleischer A. (1927-1930): Přehled brouků fauny Československé republiky, [Beetles (Coleoptera) of the Czech Republic]. 485 pp., Mor. Mus. Zemské, Brno.

Laibner S. (2000): Elateridae of the Czech and Slovak Republics. Elateridae České a Slovenské republiky. (in Czech and English). 292 pp., Kabourek, Zlín.

Löbl I. \& Smetana A. (eds) 2003: Catalogue of Palaearctic Coleoptera, Vol. 1: Archostemata - Myxophaga Adephaga. 819 pp, Apollo Books, Stenstrup.

-(eds) 2004: Catalogue of palaearctic Coleoptera, Vol. 2: Hydrophiloidea, Histeroidea, Staphylinoidea. 942 pp, Aplollo Books, Stenstrup.

-(eds) 2006: Catalogue of Palaearctic Coleoptera, Vol. 3: Scarabaeoidea - Scirtoidea - Dasciloidea Buprestoidea - Byrrhoidea. 690 pp, Apollo Books, Stenstrup.

-(eds) 2007: Catalogue of Palaearctic Coleoptera, Vol. 4: Elateroidea - Derodontoidea - Bostrichoidea Lymexyloidea - Cleroidea - Cucujoidea. 935 pp, Apollo Books, Stenstrup.

Merta L. (in litt.): Inventarizační průzkum NPR Zástudánčí z oboru hydrobiologie. (Závěrečná zpráva), [Hydrobiology Inventory study of the Zástudánčí National Nature Reserve]. Unpubl. manuscript, deposited at the Administration of the Litovelské Pomoraví PLA, 2004: 11 pp.

Nakládal O. \& Krásenský P. (2009): Faunistic records from Czech Republic - 273. Coleoptera: Staphylinidae: Aleocharinae. Klapalekiana, 45 (1-2): 32.

Pruner L. \& Míka P. (1996): Seznam obcí a jejich částí v České republice s čísly mapových polí pro sítové mapování fauny. (List of settlements in the Czech Republic with associated map field codes for faunistic grid mapping system). Klapalekiana 32 (Suppl.): 1-115.

Šafář J. et al. (2003): Olomoucko [Olomouc region]. In: Mackovčin P., Sedláček M. (eds.): Chráněná území ČR, svazek VI, [Protected areas of the Czech Republic, volume VI]. Agentura ochrany př́rody a krajiny ČR a EkoCentrum Brno, Praha, 456 pp.

Vávra J. (1995): Faunistic records from the Czech Republic - 27. Coleoptera: Cerophytidae. Klapalekiana, 31 : 70.

- (2005): Cerophytidae, p 474. In: Farkač J., Král D. \& Škorpík M. (eds.): 2005: Červený seznam ohrožených druhů České Republiky - Bezobratlí. (Red list of threatened species in the Czech Republic - Invertebrates). AOPK, Praha, 758 pp.

\section{Výsledky průzkumu brouků (Coleoptera) NPR Zástudánčí (střední Morava) provede- ného $v$ roce $2008-1$. část}

V NPR Zástudánčí, která se nachází na střední Moravě jihovýchodně od města Tovačov byl v roce 2008 proveden inventarizační průzkum brouků (Coleoptera). S cílem získat maximální druhovou diverzitu zkoumaného území bylo různými metodami odchyceno celkem 3585 exemplářů. Odychycené exempláře představují 857 druhů ze 64 čeledí. Příspěvek prezentuje faunistická data k 2191 exemplářỏm, které př̌edstavují 456 druhů z 23 čeledí.

Z celkového počtu zaznamenaných druhů je 1 druh zařazen do soustavy NATURA 2000, dle př. č.III. vyhl. MŽP ČR č. 395/1992 Sb. bylo nalezeno 5 ohrožených druhů, dle Červeného seznamu (Farkač et al. 2005) (dále jen ČS) 4 druhy kriticky ohrožené, 15 druhů ohrožených, 15 téměř ohrožených a 32 zarnitelných.

Většina zajímavých druhů (řazených do systému NATURA 2000, do př. č.III. vyhl. MŽP ČR č. 395/1992 Sb. a do ČS) je vázána na staré porosty či jednotlivé staré stromy, čili velmi stará sukcesní stádia lesa, nebo naopak na velmi mladá sukcesní stádia typu př́ibřežních štěrkopískových lavic při březích řeky Moravy. Od tohoto by se měl odvíjet i management ochrany přírody. Zatímco prríbřežní porosty nejsou při zachování přirozené dynamiky řeky ohroženy, staré stromy při severozápadní hranici NPR (viz. obr. 1), rostoucí na hrázi (mimo NPR) mohou být v budoucnu ohroženy př́mou likvidací. Přitom právě tato hráz je z hlediska chrany prŕrody velmi cenná a zasluhuje zválštní pozornost. 


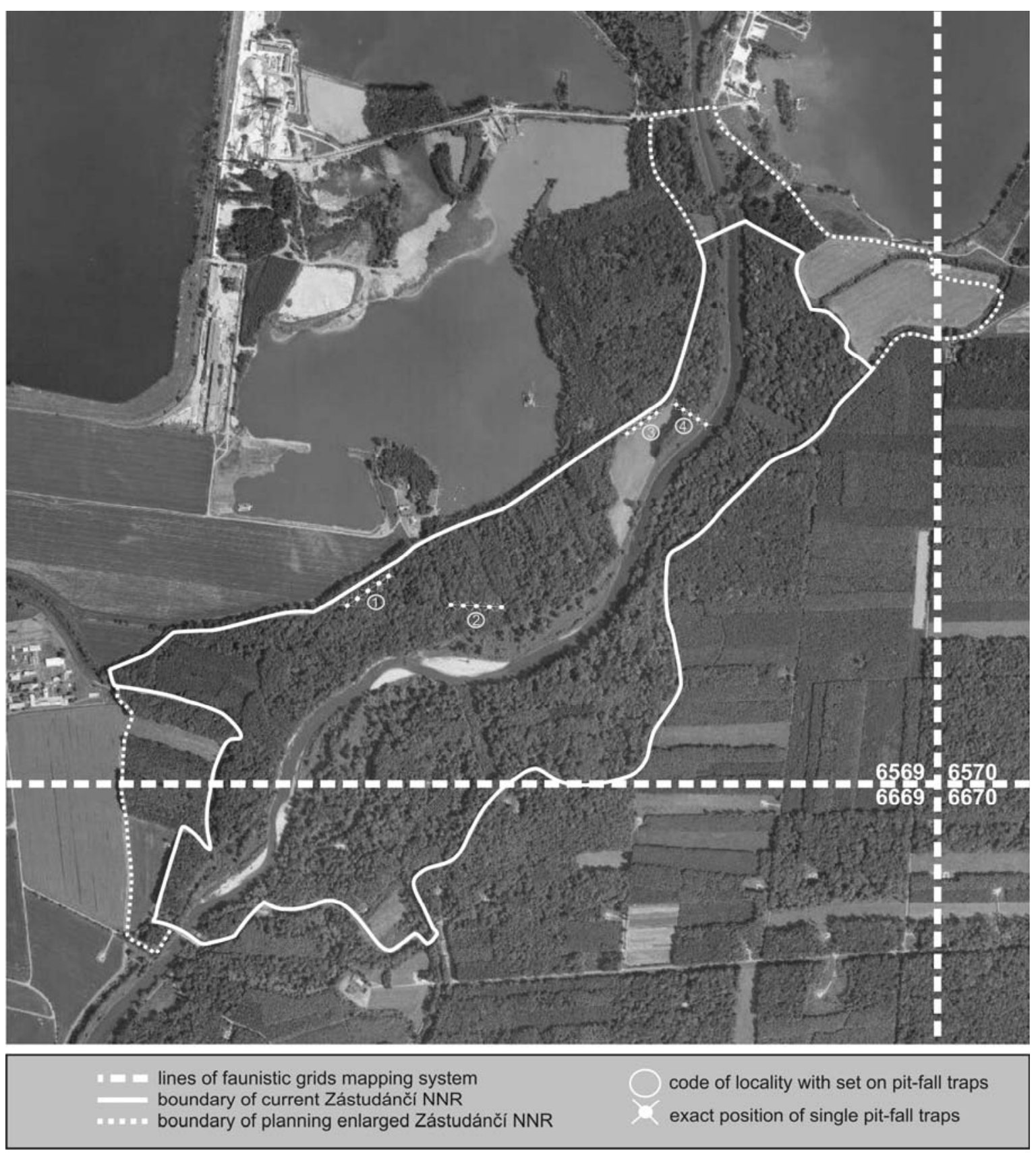

Fig 1:- Map of the survey area.

Author's address: Oto Nakládal, Department of Forest Protection and Game Management, Faculty of Forestry and Wood Sciences, Czech University of Life Sciences Prague, Kamýcká 129, CZ-165 21 Praha 6 - Suchdol, Czech Republic; e-mail: nakladal@fld.czu.cz 